\title{
China as an Integrated Area?
}

\author{
Sandra Poncet \\ Université Paris 1 \\ CEPII

\section{Jean Barthélemy} \\ Paris-Jourdan Sciences Economiques
}

\begin{abstract}
This paper documents business cycles' fluctuations in Chinese provinces. China's de facto federalism, within a context of great geographical and economic diversity, suggests the great importance of policy coordination between provinces. We investigate the role of various potential determinants of provincial business cycles' synchronization. We consider exogenous factors like distance and size as well as policy factors such as industrial, fiscal and trade policy. Results emphasize the benefits of domestic trade and local fiscal policy rationalization on the stabilization of output fluctuations. However, they also identify international trade and local economic policy heterogeneity as growing centrifugal forces.
\end{abstract}

- JEL Classification: E32, F41, E63

- Key Words: China, business cycles' co-movement, specialization, trade

\section{Introduction}

This study aims at investigating the determinants of business cycles' synchronization through a careful study of cross-regional experience within a

\footnotetext{
*Corresponding address: Sandra Poncet : Centre d'Economie de la Sorbonne (CES) Universite Paris 1 and CEPII, Maison des Sciences Economiques Bureau 405, 106 boulevard de l'Hospital, 75013 Paris, France. Tel: 01536555 54, Fax: 01536555 54, e-mail: sandra.poncet@univ-paris1. fr. Jean Barthelemy : Paris-Jourdan Sciences Economiques 
single country. We argue that the regional dimension is worth exploring because, as subunits within pre-existing currency and economic unions, regions are natural benchmarks to examine adjustment patterns to asymmetric shocks. They may also provide relevant insights on the implications of international macroeconomic coordination schemes.

We choose China as the focus of our study. This country is characterized by a de facto federalism as many regulatory responsibilities, firms as well as economic and financial powers were placed in the hands of the 30 provincial governments (Zhang and Zou, 1998). The analysis of the dynamics of business cycles' synchronization will help respond to the concerns expressed recently about whether Chinese economic reforms were successful or not in promoting domestic market integration (Young, 2000). Looking at business cycle co-fluctuation between Chinese provinces will indeed help to infer about the degree of their economic integration. ${ }^{1}$ More precisely, we investigate if business cycle synchronization proceeds in a homogenous manner across Chinese provinces or, conversely, if non-linearity exists in the correlation of regional activities in China. Assessing the business cycle co-movements of Chinese provinces has a very important implication for the evaluation of the costs associated with the current common currency within the country. This study provides a primary indication of whether Chinese provinces fulfill the condition established in the traditional literature on optimal currency areas (Mundell, 1961), under which the loss of macroeconomic flexibility associated with a common currency is small. This loss relates to the cost of processing the necessary adjustments in case of asymmetric shocks. When asymmetric macroeconomic shocks occur across the member countries, monetary policy cannot be tailored to an individual economy's particular disturbances. Hence, it is less costly for the economies to form a common currency if the business cycles' are synchronized across countries (Shin and Wang, 2004).

China's vast population and territory, as well as its geographical and economic diversity, suggest the great importance of policy coordination between the central

${ }^{1}$ As suggested by an anonymous referee, the issue of business cycles' synchronization relates to that of GDP convergence. Numerous papers have tested GDP convergence between Chinese provinces relying on different methods such as the Markov transition matrix methodology (Sakamoto and Islam, 2008) or panel unit root test (Narayan, 2008). While they fail to reach a clear consensus, they suggest possible convergence of income between Chinese provinces (Narayan, 2008; Chen and Fleisher, 1996; Jian et al., 1996). While we feel that these tests on the degree of convergence among the regions' GDP are valuable and provide complementary information on a related topic, we do believe that they lie out of the scope of this paper and that interested readers can find them in papers entirely devoted to the testing of GDP convergence. 
and sub-national governments. This issue is all the more relevant given that over the past 20 years China has made substantial efforts to decentralize its formerly autocratic fiscal and economic management system. The objective of this decentralization was to increase the local governments' responsibility for local economic development and their autonomy in carrying out fiscal functions to achieve this goal. ${ }^{2}$ However, while fiscal and economic decentralization promoted growth in many regions, it also brought many unintended problems, including rising local government deficits, weakening macroeconomic management, and increasing regional disparities (Ma and Norregaard, 1998).

We address two main questions. First, using simple correlations of output to measure business cycle synchronization, we ask what the degree is of business cycles' synchronization within China? Two related issues are firstly how it has evolved over the course of the reform process and how it differs across China? Second, what determines the extent to which business cycles' co-fluctuate between two Chinese provinces?

Byström et al. (2005) suggest that China probably is more of an optimum currency area than first expected. Our study intends to investigate the driving forces behind economic cohesion, measured by the synchronization of business cycles'. We specifically pay attention to the impact of provincial differences in terms of industrial, fiscal, monetary and trade policies. We find that overall the decentralization of economic authority to provincial governments is associated with lower economic cohesion. Notably, we estimate that over the period from 2001 to 2004, a one standard deviation increase in fiscal divergence between a pair of provinces induces a $10 \%$ reduction in their business cycles' correlation. This effect is both statistically and economically significant.

Our empirical strategy relies on monthly output data for 30 Chinese provinces over the period from 1991 to 2004 for which monthly data are available. First, we remove seasonal cycles' thanks to the US Census Bureau's X12 seasonal adjustment program. Second, we de-trend the data using the Hodrick and Prescott (1997) filter, a conventional procedure in the literature. We then calculate the correlation of the resulting cyclical components. We investigate the magnitude and determinants of cross-province correlations. We explicitly assess the role of four main factors: production specialization dissimilarity, local policy coordination, domestic integration and differences in terms of engagement in international trade.

${ }^{2}$ Reforms also intended to preserve an adequate degree of fiscal control for the central government. 
We consider the sensitivity of these relationships to the inclusion of justifiable controls and we investigate in detail possible sources of endogeneity. This analysis of business cycles' correlation is interesting to us since it should help us to deduce some policy implications as to how to promote deeper integration between Chinese provinces.

The paper is organized as follows. Section II provides some stylized facts on economic decentralization and policy coordination in China. Section III presents the empirical methodology and the data used to investigate business synchronization patterns in China. Section IV reports our main empirical findings on the determinants of business cycles' correlation between Chinese provinces. The final section concludes our findings.

\section{Economic Decentralization and Policy Coordination in China}

China's duplicative domestic market is referred to as cellularized along the provincial borders (Donnithorne, 1972) due to its horizontal principle of economic management. It is traditionally based on a territorial or regional planning where local authorities govern most economic activities in one geographic region across different economic sectors. The decentralization reforms initiated in 1980 reinforced China's de facto economic federalism as greater fiscal, economic and regulatory responsibilities as well as the ownership of firms were devolved to provincial governments. Although the central government still maintained the responsibility for defining the fiscal system so that the rate of taxation and the fiscal bases remained uniform throughout China, the administration and collection of taxes was initially devolved to the provinces on a large scale. From 1979 to 1993, the central fiscal power gradually weakened: the central government's share of expenditures (after revenue-sharing) declined from 51 percent to 28 percent (Ma and Norregaard, 1998).

The 1994 reform aimed at stopping this evolution with the intention of reinstating a greater degree of fiscal centralization. The fiscal liberty of the provinces clearly weakened, as they could no longer establish fiscal contracts with the companies and accord them exonerations as an instrument of their industrial policy or as a recompense for investment in infrastructure. Moreover, in line with the initial objective of the reform, their share of fiscal revenues declined to the benefit of the central government. Nevertheless, several features suggest that the reform concretely reinforced the fiscal autonomy of the provinces (Hua and 
Guillaumont, 2004). Notably the new law indeed leaves the provinces discretionary power in the administration of these taxes, as it is very difficult for the central administration to control an activity as vast as local fiscal administration (Balh, 1999). Moreover since provinces entirely benefit from the revenues that they collect themselves, they are in a position to adapt their collection effort to the desired level of their fiscal expenditure. This autonomy manifests itself in the extremely different fiscal effort across provinces.

The key institutional feature behind the divergence between provincial interests and the lack of policy coordination in China is the continuing role of local governments as owners and managers of a significant part of the local economic base. Most authors recognize that distortions remaining in the beginning of the 1990s, most notably disproportions between "basic" raw materials sectors and "processing" sectors, justified the divergence of provincial objectives and policies across China. There is however a debate on the evolution since the mid 1990s. At one end of the spectrum, Young (2000) makes the extreme assertion that, over the past 20 years of economic reform, China has evolved into "a fragmented internal market with fiefdoms controlled by local officials". On the other end of the spectrum, Naughton (2003) indicates a reduction since the mid 1990s in the forces constraining local governments and tipping them toward market intervention (such as the need to defend employment levels). The author claims that the central government's efforts to impose a harder budget constraint on the state banking system have consequently made it more costly for local governments to attempt to countermand market outcomes. The analysis of the dynamics of business cycles' synchronization will help determine whether the devolution of discretionary economic and fiscal power in the hands of provinces translated into greater economic cohesion in China. This question relates to the anti or pro-cyclical nature of provincial policies: does policy heterogeneity help to offset asymmetric shocks or is it in itself a source of shocks? Furthermore, it will indicate whether the deepening of economic reforms as well as the fiscal recentralization attempts in the mid 1990s were successful or not in promoting domestic market integration.

\section{Data and Empirical Methodology}

\section{A. Evidence from Raw Data}

We use provincial gross output value of industry reported every month. ${ }^{3}$ by the 
National Bureau of Statistics (NBS) from the third month of 1991 to the first month of 2005. Output figures are expressed in 1990 constant price. They cover all the 30 Chinese provinces ${ }^{4}$ except Tibet. This province is dropped from our sample due to much missing data. ${ }^{5}$

We start with a direct comparison of business cycles' synchronization between Chinese provinces. First, seasonal patterns are removed by the US Census Bureau's X12 seasonal adjustment program. Second, the cyclical component of output fluctuations is isolated by filtering our data on regional activity through the Hodrick and Prescott (1997)'s filter. ${ }^{6}$ Finally, the degree of synchronization of business cycles' is measured using estimated correlation coefficients on the detrended provincial output $y$.

Formally, let $\hat{\rho}_{i j}$ be the estimated correlation between the cyclical components of regions $i$ and $j, y_{j}$ and $y_{j}$, respectively: $\hat{\rho_{i j}}=\operatorname{cov}\left(y_{i}, y_{j}\right) / \sqrt{\operatorname{var}\left(y_{i}\right) \operatorname{var}\left(y_{j}\right)}$.

Bilateral correlations are computed over the thirteen-year period of the dataset (1991-2004). We also split the sample into three sub-periods: 1991-1995, 19962000 and 2000-2004 to grasp insight into how economic cohesion evolved over the reform progress. Those three sub-periods correspond to three successive steps of the economic reforms and exactly match the Eighth, Ninth and Tenth Five-year plans respectively.

The Eighth Five-year Plan (1991-1995) corresponds to the first strides on the road to socialism with Chinese characteristics. The important speech by Deng Xiaoping in 1992 marked the beginning of a new stage for reform in terms of opening up and modernizing. The "industrial policy" of the first half of the 1990s focused overwhelmingly on energy, steel, and other "basic industries". Infrastructure development was also at the top of the agenda in order to ease shortages and bottlenecks. By the mid-1990s, though, this economic environment changed. Economic reform reduced the demand for intermediate goods, while

\footnotetext{
${ }^{3}$ While data on annual GDP or output for all Chinese provinces are available for the period 1952-2004, monthly data are available only from 1990 onwards.

${ }^{4}$ Chongqing data were consolidated with those of Sichuan in order to ensure time consistency despite the fact that this municipality was granted the provincial status in 1997. Data sources are detailed in the appendix.

${ }^{5}$ Relying on employment data, we find out that Tibet business cycles are negatively correlated with those of other provinces. This suggests that Tibet is a complete outlier. Its exclusion from the sample would therefore be required even though data would have been available.

${ }^{6}$ In applying the filter, we choose the parameters following those recommended by the literature. In the case of the HP filter, we have set the smoothing parameter lambda at 14400 for monthly data (Ravn and Uhlig, 2002).
} 
supply increased.

The Ninth Five-year Plan (1996-2000) consequently focused more on the development of pillar industries such as machinery, petroleum processing, raw chemical materials and construction industries to promote overall economic development (Lu, 2002). Pillar industries were expected to have high-income elasticity, significant technological (learning by doing) content, and spillover effects in economic development. Local governments put in charge of carrying out their own planning exercises, responded enthusiastically and took investment decisions that sharply increased the new capacity under construction. Naughton (2003) argues that the result has been something just short of disastrous, with massive over-capacity in a range of "pillar industries", especially building materials, metallurgy, petrochemistry and automobile industry.

The year 2001 marked the entry of China into the WTO and locked China into the path of reform in terms of financial liberalization and marketization. The tenth Five Year Plan (2001-2005) promotes further engagement in international integration. At the same time, however, rising disparities in line with great economic performance heterogeneity induce authorities to mitigate their preferential treatment towards the Coast and foster economic development of the interior provinces.

Tables 1 and 2 report some basic correlation results. Table 1 gives the average correlation for each province for each sub-period while Table 2 differentiates average correlation according to the traditional sub-division into two regions: Coast and Interior. This decomposition into coastal and interior provinces is one commonly used in official publication and still guides economic and industrial policy planning. This subdivision draws from the recognition of China's regional dualistic development structure and geographic, economic and administrative coastal-interior cleavages (Yang, 1997). In China, the coastal region has both a more developed transport infrastructure and a higher engagement in economic liberalization and restructuring. Conversely, interior provinces suffered greatly from distorted central policies (undervalued raw materials prices, preferential taxation for the coast, investment and reforms biased in favor of coastal regions). These depressed interior provinces resorted to autarkical policies (import substitution and protectionism) to make up for the perceived unfairness and developed their own processing industries in the shelter of trade restrictions over the end of 1980s and beginning of 1990s. Figure 1 in the appendix displays the distribution of bilateral business cycle correlations to help the reader better 
Table 1. Average Business Cycles' Synchronization by Province

\begin{tabular}{|c|c|c|c|c|}
\hline Output Correlation & 1991-2004 & 1991-1995 & $1996-2000$ & 2001-2004 \\
\hline \multicolumn{5}{|c|}{ Coastal Regions } \\
\hline Beijing & 0.42 & 0.39 & 0.23 & 0.58 \\
\hline Tianjin & 0.06 & 0.39 & -0.27 & 0.41 \\
\hline Hebei & 0.55 & 0.49 & 0.37 & 0.70 \\
\hline Liaoning & 0.53 & 0.47 & 0.35 & 0.70 \\
\hline Shanghai & 0.19 & 0.40 & -0.17 & 0.39 \\
\hline Jiangsu & 0.41 & 0.33 & 0.12 & 0.70 \\
\hline Zhejiang & 0.36 & 0.47 & 0.11 & 0.49 \\
\hline Fujian & 0.33 & 0.45 & 0.18 & 0.51 \\
\hline Shandong & 0.50 & 0.39 & 0.23 & 0.68 \\
\hline Guangdong & 0.21 & 0.53 & -0.12 & 0.35 \\
\hline Guangxi & 0.37 & 0.42 & 0.32 & 0.37 \\
\hline Hainan & 0.27 & 0.21 & 0.17 & 0.47 \\
\hline \multicolumn{5}{|c|}{ Interior Regions } \\
\hline Shanxi & 0.43 & 0.20 & 0.17 & 0.64 \\
\hline Nei Monggol & 0.52 & 0.42 & 0.32 & 0.69 \\
\hline Jilin & 0.38 & 0.31 & 0.29 & 0.51 \\
\hline Heilongjiang & 0.49 & 0.34 & 0.38 & 0.61 \\
\hline Anhui & 0.42 & 0.55 & 0.34 & 0.67 \\
\hline Jiangxi & 0.46 & 0.43 & 0.26 & 0.60 \\
\hline Henan & 0.46 & 0.49 & 0.22 & 0.59 \\
\hline Hubei & 0.49 & 0.51 & 0.29 & 0.67 \\
\hline Hunan & 0.52 & 0.48 & 0.36 & 0.70 \\
\hline Guizhou & 0.46 & 0.32 & 0.33 & 0.68 \\
\hline Yunnan & 0.30 & 0.47 & 0.04 & 0.34 \\
\hline Shaanxi & 0.52 & 0.50 & 0.29 & 0.70 \\
\hline Gansu & 0.40 & 0.27 & 0.23 & 0.50 \\
\hline Qinghai & 0.30 & -0.02 & 0.04 & 0.47 \\
\hline Ningxia & 0.46 & 0.34 & 0.24 & 0.61 \\
\hline Xinjiang & 0.42 & 0.22 & 0.26 & 0.53 \\
\hline Sichuan & 0.45 & 0.56 & 0.38 & 0.49 \\
\hline
\end{tabular}

Source: Authors' computation.

visualize our explained variable.

Results emphasize a decrease in the average level of cycle synchronization between 1991-1995 and 1996-2000 followed by an increase over the following period. It shrinks by nearly $50 \%$ between the first two periods from 0.39 to 0.20 and it rose by nearly $185 \%$ to reach 0.57 over the 2001-2004 period. The decrease 
Table 2. Average Output Correlation by Group of Regions

\begin{tabular}{|c|c|c|c|c|c|c|}
\hline & \multirow{2}{*}{$\begin{array}{c}1991-1995 \\
\text { I }\end{array}$} & \multicolumn{2}{|c|}{$1996-2000$} & \multicolumn{3}{|c|}{ 2001-2005 } \\
\hline & & II & Evolution I-II & III & Evolution II-III & Evolution I-III \\
\hline $\begin{array}{l}\text { Coastal } \\
\text { Region } \\
(55 \text { obs. })\end{array}$ & $\begin{array}{c}0.541 \\
(0.0237)\end{array}$ & $\begin{array}{c}0.115 \\
(0.0428)\end{array}$ & $-78.79 \% * * *$ & $\begin{array}{c}0.563 \\
(0.0305)\end{array}$ & $390.38 \% * * *$ & $4.01 \%$ \\
\hline $\begin{array}{l}\text { Interior } \\
\text { Region } \\
\text { (136 obs.) }\end{array}$ & $\begin{array}{c}0.386 \\
(0.0191)\end{array}$ & $\begin{array}{c}0.343 \\
(0.0224)\end{array}$ & $-11.08 \% *$ & $\begin{array}{c}0.671 \\
(0.0162)\end{array}$ & $95.37 \% * * *$ & $73.72 \% * * *$ \\
\hline $\begin{array}{l}\text { Interior- } \\
\text { Coast } \\
\text { (187 obs.) }\end{array}$ & $\begin{array}{c}0.368 \\
(0.0174)\end{array}$ & $\begin{array}{c}0.129 \\
(0.0268)\end{array}$ & $-64.81 \% * * *$ & $\begin{array}{c}0.517 \\
(0.0204)\end{array}$ & $299.29 \% * * *$ & $40.49 \% * * *$ \\
\hline $\begin{array}{c}\text { Global } \\
\text { Average } \\
\text { (378 obs.) }\end{array}$ & $\begin{array}{c}0.400 \\
(0.0119)\end{array}$ & $\begin{array}{c}0.204 \\
(0.0175)\end{array}$ & $-48.90 \% * * *$ & $\begin{array}{c}0.579 \\
(0.01296)\end{array}$ & $183.45 \% * * *$ & $44.85 \% * * *$ \\
\hline
\end{tabular}

Robust standard errors in parentheses

$*, * *$ and $* * *$ denote significance of the difference at $10 \%, 5 \%$ and $1 \%$ confidence level respectively.

\section{International context}

EU and US:1981-1997

France/Germany: 1983-1996
Average within country 0.64

Average cross-country 0.4 Average

Average within country 0.74

Average cross-country 0.43 
Figure 1. Business Cycles Correlation Histograms
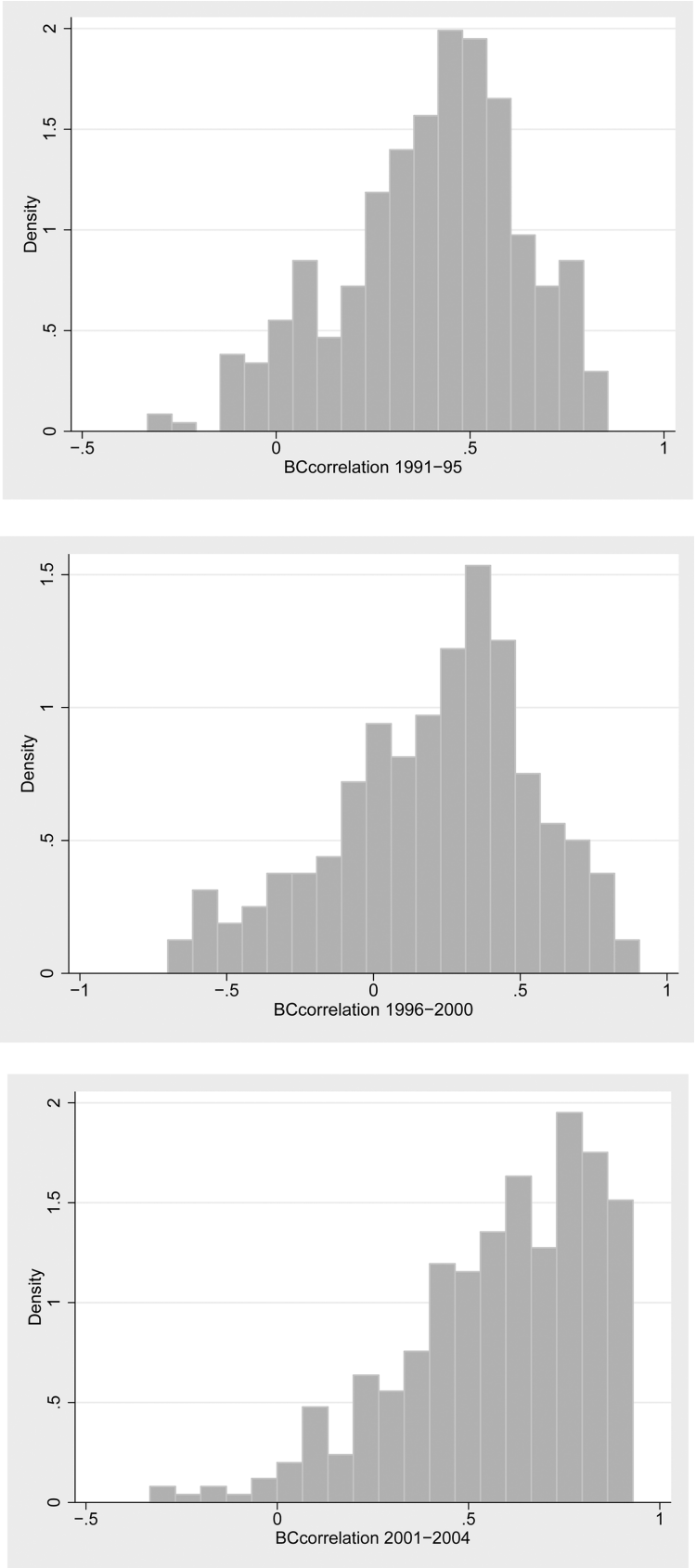
in business cycle correlation over the 1990s appears to be a general evolution. However it is clearly driven by the specific cases of Tianjin, Shanghai and Guangdong. The business cycle correlation of these provinces with the rest of the country drops from a positive and relatively high value to a negative one throughout the decade. This pattern relates to the rising heterogeneity between those coastal provinces that engaged boldly in the process of enterprise reform, privatization and marketization and those crippled by the weight of inefficient and out-of-date heavy industries. Some provinces, like Shanghai or Guangdong, enjoyed a strong growth in comparison to other coastal provinces, which suffered from economic stagnation over the second sub-period.

We anticipate internationally integrated and liberalized coastal provinces' activities to be determined by specific features and as such to be relatively independent from the national business cycle. We need to verify whether this pattern manifests itself in the existence of a border effect, that is, a significantly lower business cycle correlation between the coast and the rest of the country. However, it is possible that highly localized rapid economic growth in a few coastal provinces makes it inappropriate to consider the coast as a homogeneous group. As a matter of fact, rapid growth occurred in a heterogeneous fashion across coastal provinces. As such, localized successes amid more modest progress may materialize in lower business cycle correlation between provinces acting as free agents and provinces that are more in line with the national trend. Lower correlation may be expected from within the coastal region than between Interior and Coastal provinces as well as between Interior provinces.

This evolution is reversed towards the end of the $20^{\text {th }}$ century. Average business cycles' correlation within China climbed from a rather low level in the beginning of the century to a figure, over the period from 2001 to 2005, that is almost comparable to that existing between regions within the US as measured by Clark and Van Wincoop (2001). These authors find that the average within country GDP correlation was around 0.64 for the United States and European Union against 0.40 for the average cross-country correlation over 1981-1997. These figures can also be compared to those for France and Germany: average correlation within country is found to be 0.74 against 0.43 for the average cross-country correlation over the period 1983-1996.

Thus from a rather low level of business cycles' synchronization at the beginning of the 1990s, China evolved to a level quite similar to that of developed economies at the beginning of the 21th century. The investigation of cycles' 
synchronization determinants in China will shed light on the forces at work behind the successive drop and increase of business cycle synchronization in China. It will help to identify centrifugal forces that may threaten China's economic cohesion as well as centripetal forces that central authorities should promote.

\section{B. Estimation Method}

In order to investigate the determinants of cycle's synchronization in China, we regress bilateral correlations in business cycles' across Chinese provinces based on the following equation:

$$
\hat{\rho_{i j}}=\alpha+\beta X_{i j}+\gamma{\text { Dis } \tan c e_{i j}}+\text { SAdjacency }_{i j}+\kappa \text { Size }_{i j}+\lambda W_{i j}+\varepsilon_{i j}
$$

where $X_{i j}$ encompass variables intended to test for potential spatial discontinuities in China and include determinants of business cycles' synchronization other than those relating to distance, adjacency and size. We follow Frankel and Rose (1998), Imbs (2003), and Calderón et al. (2003) in using White (1980) heteroskedasticityconsistent standard errors for hypothesis testing purposes. As concerns about potential endogeneity will rise for some of the determinants we intend to investigate, we perform Durbin-Wu-Hausman test ${ }^{7}$ and proceed with two-stagesleast-squares (2SLS) estimations. This approach intends to deal with empirical biases due to two distinct problems: the reverse impact of business cycles correlation on its determinants and the potential correlation between the investigated determinants and the regression residuals which contain random shocks and other omitted factors. As such, our instrumental procedure will guarantee that our results are not significantly affected by other exogenous or endogenous mechanisms not accounted for in the estimations. ${ }^{8}$ We moreover account for the potentially complex variance-covariance structure of the residuals relying on the two-step efficient generalized method of moments (GMM) estimator. $^{9}$

First, we directly test the existence of discontinuities in terms of regional activities' correlation in China through the study of a Coast-Interior separation line. This approach draws from the recognized atypicality and marginalization of the

\footnotetext{
${ }^{7}$ Validity of instruments has been tested by Hansen $\mathrm{J}$ statistic overidentification test. ${ }^{8} \mathrm{We}$ thank an anonymous referee for raising this issue.

${ }^{9}$ As stated in Clark and Van Wincoop (2001), the fact that correlations in business cycles are measured with error may create a specific type of heteroskedasticity in the residuals of the equation that standard White corrections connot account for.
} 
Table 3. Business Cycles' Synchronization-sub-period 1991-1995

\begin{tabular}{|c|c|c|c|c|c|c|c|c|c|}
\hline & $\begin{array}{c}1 \\
\text { OLS }\end{array}$ & $\begin{array}{c}2 \\
\text { OLS }\end{array}$ & $\begin{array}{c}3 \\
\text { OLS }\end{array}$ & $\begin{array}{c}4 \\
\text { IV-GMM }\end{array}$ & $\begin{array}{c}5 \\
\text { IV-GMM }\end{array}$ & $\begin{array}{c}6 \\
\text { IV-GMM }\end{array}$ & $\begin{array}{c}7 \\
\text { IV-GMM }\end{array}$ & $\begin{array}{c}8 \\
\text { IV-GMM }\end{array}$ & $\begin{array}{c}9 \\
\text { IV-GMM }\end{array}$ \\
\hline Intra Coast & $\begin{array}{c}0.155^{* * * *} \\
(0.030)\end{array}$ & $\begin{array}{c}0.121^{* * * *} \\
(0.029)\end{array}$ & $\begin{array}{c}0.138^{* * * *} \\
(0.030)\end{array}$ & $\begin{array}{c}0.092^{* * * *} \\
(0.028)\end{array}$ & $\begin{array}{c}0.109^{* * * *} \\
(0.031)\end{array}$ & $\begin{array}{c}0.080^{* * * *} \\
(0.031)\end{array}$ & $\begin{array}{l}0.058^{*} \\
(0.035)\end{array}$ & $\begin{array}{c}0.042 \\
(0.032)\end{array}$ & $\begin{array}{c}0.025 \\
(0.035)\end{array}$ \\
\hline Coast-Interior Border & $\begin{array}{l}-0.018 \\
(0.026)\end{array}$ & $\begin{array}{l}-0.028 \\
(0.022)\end{array}$ & $\begin{array}{c}0.015 \\
(0.023)\end{array}$ & $\begin{array}{l}-0.004 \\
(0.022)\end{array}$ & $\begin{array}{l}-0.002 \\
(0.022)\end{array}$ & $\begin{array}{c}0.012 \\
(0.024)\end{array}$ & $\begin{array}{c}0.004 \\
(0.025)\end{array}$ & $\begin{array}{l}-0.008 \\
(0.025)\end{array}$ & $\begin{array}{l}-0.014 \\
(0.026)\end{array}$ \\
\hline Size (1) & & $\begin{array}{c}0.556 * * * \\
(0.057)\end{array}$ & $\begin{array}{c}0.452 * * * \\
(0.060)\end{array}$ & $\begin{array}{c}0.327 * * * \\
(0.060)\end{array}$ & $\begin{array}{c}0.330^{* * * *} \\
(0.064)\end{array}$ & $\begin{array}{c}0.304 * * * \\
(0.083)\end{array}$ & $\begin{array}{c}0.289^{* * * *} \\
(0.084)\end{array}$ & $\begin{array}{c}0.216^{* * * *} \\
(0.070)\end{array}$ & $\begin{array}{c}0.209 * * * \\
(0.070)\end{array}$ \\
\hline Distance (2) & & $\begin{array}{c}-0.368 * * * \\
(0.106)\end{array}$ & $\begin{array}{c}-0.259 * * \\
(0.106)\end{array}$ & $\begin{array}{c}-0.226^{* *} \\
(0.101)\end{array}$ & $\begin{array}{l}-0.164 \\
(0.117)\end{array}$ & $\begin{array}{c}-0.566 * * * \\
(0.150)\end{array}$ & $\begin{array}{c}-0.460 * * * \\
(0.161)\end{array}$ & $\begin{array}{c}-0.579 * * * \\
(0.146)\end{array}$ & $\begin{array}{c}-0.474 * * * \\
(0.160)\end{array}$ \\
\hline Adjacency & & $\begin{array}{c}0.008 \\
(0.030)\end{array}$ & $\begin{array}{c}0.004 \\
(0.030)\end{array}$ & $\begin{array}{c}0.012 \\
(0.027)\end{array}$ & $\begin{array}{c}0.014 \\
(0.027)\end{array}$ & $\begin{array}{l}0.096^{*} \\
(0.058)\end{array}$ & $\begin{array}{l}0.106^{*} \\
(0.058)\end{array}$ & $\begin{array}{l}0.117 * * \\
(0.048)\end{array}$ & $\begin{array}{c}0.125^{* * * *} \\
(0.048)\end{array}$ \\
\hline Dissimilarity of Production Structure & & & $\begin{array}{c}-0.243 * * * \\
(0.068)\end{array}$ & $\begin{array}{c}-0.161 * * \\
(0.070)\end{array}$ & $\begin{array}{c}-0.144 * \\
(0.075)\end{array}$ & $\begin{array}{l}-0.090 \\
(0.116)\end{array}$ & $\begin{array}{l}-0.075 \\
(0.118)\end{array}$ & $\begin{array}{l}-0.111 \\
(0.095)\end{array}$ & $\begin{array}{l}-0.093 \\
(0.098)\end{array}$ \\
\hline $\begin{array}{l}\text { A_Dissimilarity of Local Policy } \\
\text { (standard deviation of public deficit) }\end{array}$ & & & & $\begin{array}{c}-3.037 * * * \\
(0.723)\end{array}$ & $\begin{array}{l}-2.937 * * * \\
(0.808)\end{array}$ & $\begin{array}{c}-4.483 * * \\
(1.782)\end{array}$ & $\begin{array}{l}-4.748 * * * \\
(1.806)\end{array}$ & $\begin{array}{l}-5.568 * * * \\
(1.247)\end{array}$ & $\begin{array}{l}-5.743 * * * \\
(1.264)\end{array}$ \\
\hline $\begin{array}{l}\text { B_Dissimilarity of Local Policy } \\
\text { (Consumer Price Index differential) }\end{array}$ & & & & & $\begin{array}{l}-0.015 \\
(0.017)\end{array}$ & $\begin{array}{c}0.022 \\
(0.017)\end{array}$ & $\begin{array}{c}0.027 \\
(0.017)\end{array}$ & $\begin{array}{c}0.008 \\
(0.013)\end{array}$ & $\begin{array}{c}0.014 \\
(0.014)\end{array}$ \\
\hline C_ Railways Freight Traffic (2) & & & & & & $\begin{array}{l}-0.783^{*} \\
(0.437)\end{array}$ & $\begin{array}{l}-0.787^{*} \\
(0.436)\end{array}$ & $\begin{array}{c}-0.909 * * \\
(0.368)\end{array}$ & $\begin{array}{c}-0.902^{* *} \\
(0.368)\end{array}$ \\
\hline D_ Dissimilarity of Trade Intensity & & & & & & & & $\begin{array}{c}0.247 * * * \\
(0.085)\end{array}$ & $\begin{array}{c}0.222 * * * \\
(0.086)\end{array}$ \\
\hline E_Dissimilarity of International Partner & & & & & & & $\begin{array}{l}-0.087^{*} \\
(0.052)\end{array}$ & & $\begin{array}{l}-0.086 \\
(0.053)\end{array}$ \\
\hline Constant & $\begin{array}{c}0.386 * * * \\
(0.019)\end{array}$ & $\begin{array}{c}0.367 * * * \\
(0.035)\end{array}$ & $\begin{array}{c}0.497 * * * \\
(0.049)\end{array}$ & $\begin{array}{c}0.565^{* * * *} \\
(0.046)\end{array}$ & $\begin{array}{c}0.567 * * * \\
(0.051)\end{array}$ & $\begin{array}{c}0.617 * * * * \\
(0.071)\end{array}$ & $\begin{array}{c}0.652 * * * * \\
(0.076)\end{array}$ & $\begin{array}{c}0.700 * * * \\
(0.059)\end{array}$ & $\begin{array}{c}0.726^{* * * *} \\
(0.062)\end{array}$ \\
\hline Observations & 378 & 378 & 378 & 378 & 378 & 378 & 378 & 378 & 378 \\
\hline $\mathrm{R}^{2}$ & 0.065 & 0.31 & 0.34 & 0.42 & 0.39 & 0.33 & 0.33 & $\begin{array}{l}0.29 \\
0.92\end{array}$ & 0.30 \\
\hline $\begin{array}{l}\text { Hansen Overindentification Test } \\
\text { List of Endogenous Variables } \\
\text { (partial } \mathrm{R}^{2} \text { of excluded instruments } \\
\text { in parentheses) }\end{array}$ & & & & $\mathrm{A}(0.43)$ & $\begin{array}{c}0.61 \\
\mathrm{~A}(0.43) \\
\quad \text { and } \\
\mathrm{B}(0.20)\end{array}$ & $\begin{array}{c}0.97 \\
\mathrm{~A}(0.43) \\
\mathrm{B}(0.25) \\
\text { and } \mathrm{C}(0.10)\end{array}$ & $\begin{array}{c}0.96 \\
\mathrm{~A}(0.39) \\
\mathrm{B}(0.24) \\
\text { and } \mathrm{C}(0.10)\end{array}$ & $\begin{array}{c}0.92 \\
\mathrm{~A}(0.44), \\
\mathrm{B}(0.36), \\
\text { and } \mathrm{C}(0.11)\end{array}$ & $\begin{array}{c}0.90 \\
\mathrm{~A}(0.42) \\
\mathrm{B}(0.35) \\
\text { and } \mathrm{C}(0.10)\end{array}$ \\
\hline
\end{tabular}

Robust standard errors in parentheses calculated as indicated by White (1980)

* significant at $10 \%$; ** significant at 5\%;*** significant at $1 \%$

(1) and (2): coefficients have been multiplied by $10^{10}$ and $10^{4}$ respectively 
Table 4. Business Cycles' Synchronization-sub-period 1996-2000

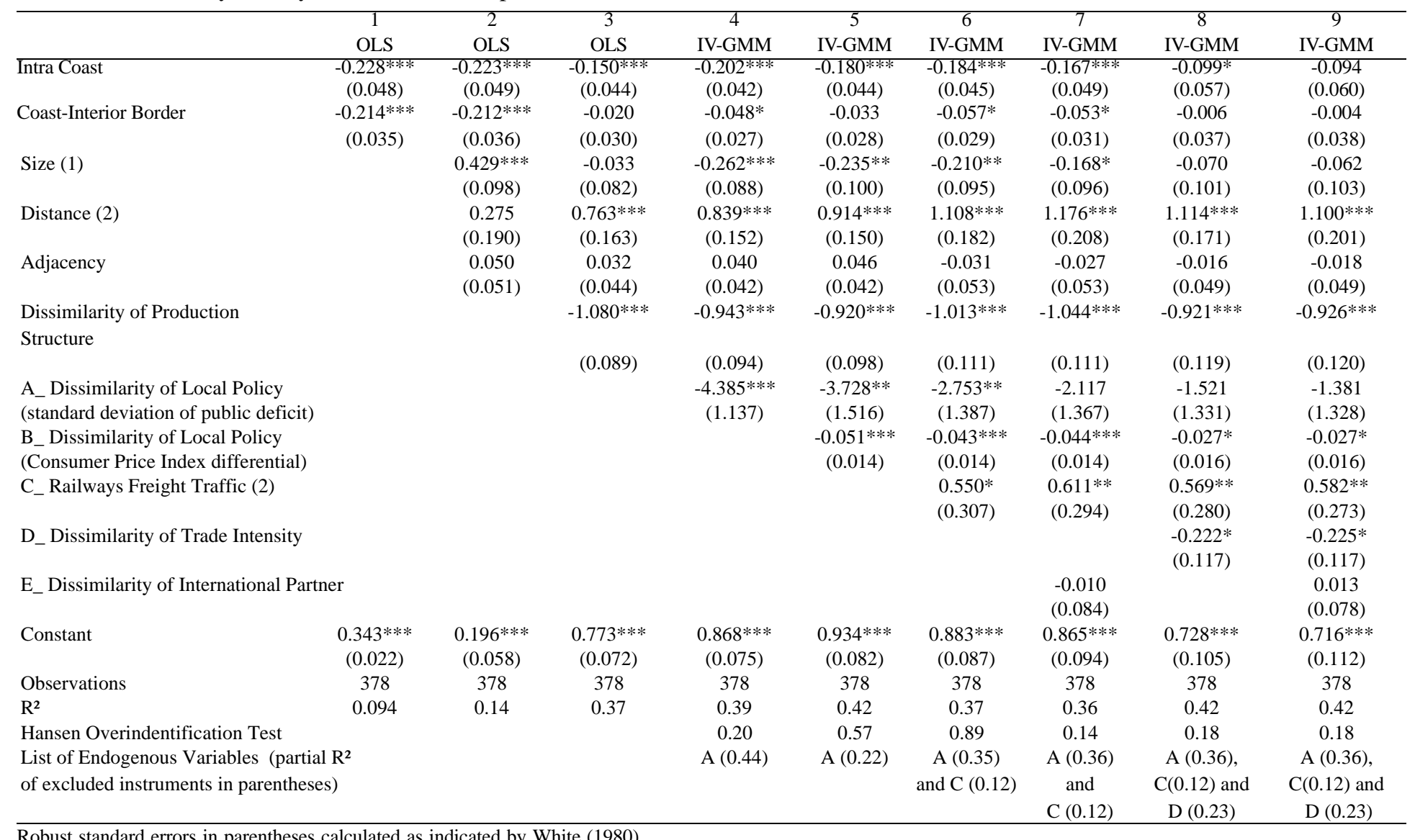

(1) and (2): coefficients have been multiplied by $10^{10}$ and $10^{4}$ respectively 
Table 5. Business Cycles' Synchronization-sub-period 2001-2004

\begin{tabular}{|c|c|c|c|c|c|c|c|c|c|}
\hline & $\begin{array}{c}1 \\
\text { OLS }\end{array}$ & $\begin{array}{c}2 \\
\text { OLS }\end{array}$ & $\begin{array}{c}3 \\
\text { OLS }\end{array}$ & $\begin{array}{c}4 \\
\text { IV-GMM }\end{array}$ & $\begin{array}{c}5 \\
\text { IV-GMM }\end{array}$ & $\begin{array}{c}6 \\
\text { IV-GMM }\end{array}$ & $\begin{array}{c}7 \\
\text { IV-GMM }\end{array}$ & $\begin{array}{c}8 \\
\text { IV-GMM }\end{array}$ & $\begin{array}{c}9 \\
\text { IV-GMM }\end{array}$ \\
\hline Intra Coast & $\begin{array}{l}-0.108^{* * * *} \\
(0.034)\end{array}$ & $\begin{array}{l}-0.147 * * * * \\
(0.033)\end{array}$ & $\begin{array}{l}-0.108^{* * * *} \\
(0.031)\end{array}$ & $\begin{array}{l}-0.136^{* * * *} \\
(0.033)\end{array}$ & $\begin{array}{l}-0.129 * * * * \\
(0.033)\end{array}$ & $\begin{array}{l}-0.108^{* * * *} \\
(0.035)\end{array}$ & $\begin{array}{c}-0.230^{* * * *} \\
(0.051)\end{array}$ & $\begin{array}{l}-0.091^{*} \\
(0.049)\end{array}$ & $\begin{array}{l}-0.159^{* * * *} \\
(0.056)\end{array}$ \\
\hline Coast-Interior Border & $\begin{array}{c}-0.153^{* * * *} \\
(0.026)\end{array}$ & $\begin{array}{c}-0.162 * * * \\
(0.026)\end{array}$ & $\begin{array}{c}-0.060^{* * *} \\
(0.028)\end{array}$ & $\begin{array}{c}-0.068^{* *} \\
(0.028)\end{array}$ & $\begin{array}{l}-0.055^{*} \\
(0.028)\end{array}$ & $\begin{array}{c}-0.102 * * * \\
(0.032)\end{array}$ & $\begin{array}{c}-0.145^{* * * *} \\
(0.036)\end{array}$ & $\begin{array}{l}-0.079^{*} \\
(0.044)\end{array}$ & $\begin{array}{l}-0.111 * * \\
(0.045)\end{array}$ \\
\hline Size (1) & & $\begin{array}{c}0.076 \\
(0.078)\end{array}$ & $\begin{array}{c}-0.171^{* *} \\
(0.084)\end{array}$ & $\begin{array}{c}-0.308 * * * \\
(0.100)\end{array}$ & $\begin{array}{c}-0.361 * * * \\
(0.102)\end{array}$ & $\begin{array}{l}-0.120 \\
(0.101)\end{array}$ & $\begin{array}{c}-0.322 * * * \\
(0.116)\end{array}$ & $\begin{array}{l}-0.110 \\
(0.095)\end{array}$ & $\begin{array}{l}-0.216^{*} \\
(0.110)\end{array}$ \\
\hline Distance (2) & & $\begin{array}{c}-0.672 * * * \\
(0.137)\end{array}$ & $\begin{array}{c}-0.411^{* * * *} \\
(0.123)\end{array}$ & $\begin{array}{c}-0.372 * * * * \\
(0.125)\end{array}$ & $\begin{array}{c}-0.332^{* * * *} \\
(0.124)\end{array}$ & $\begin{array}{l}-0.039 \\
(0.147)\end{array}$ & $\begin{array}{c}0.493 \\
(0.333)\end{array}$ & $\begin{array}{l}-0.168 \\
(0.183)\end{array}$ & $\begin{array}{c}0.592 \\
(0.371)\end{array}$ \\
\hline Adjacency & & $\begin{array}{l}-0.026 \\
(0.036)\end{array}$ & $\begin{array}{l}-0.036 \\
(0.035)\end{array}$ & $\begin{array}{l}-0.026 \\
(0.035)\end{array}$ & $\begin{array}{l}-0.049 \\
(0.035)\end{array}$ & $\begin{array}{c}-0.175^{* * * *} \\
(0.053)\end{array}$ & $\begin{array}{l}-0.085^{*} \\
(0.051)\end{array}$ & $\begin{array}{c}-0.128^{* * * *} \\
(0.050)\end{array}$ & $\begin{array}{c}-0.112^{* *} \\
(0.056)\end{array}$ \\
\hline Dissimilarity of Production Structure & & & $\begin{array}{c}-0.578 * * * \\
(0.082)\end{array}$ & $\begin{array}{c}-0.506^{* * *} \\
(0.087)\end{array}$ & $\begin{array}{c}-0.501 * * * \\
(0.084)\end{array}$ & $\begin{array}{c}-0.653 * * * \\
(0.089)\end{array}$ & $\begin{array}{c}-0.467 * * * \\
(0.096)\end{array}$ & $\begin{array}{c}-0.532 * * * \\
(0.183)\end{array}$ & $\begin{array}{c}-0.427 * * \\
(0.191)\end{array}$ \\
\hline $\begin{array}{l}\text { A_Dissimilarity of Local Policy } \\
\text { (standard deviation of public deficit) }\end{array}$ & & & & $\begin{array}{c}-2.640 * * \\
(1.225)\end{array}$ & $\begin{array}{c}-3.025 * * \\
(1.251)\end{array}$ & $\begin{array}{c}1.448 \\
(1.101)\end{array}$ & $\begin{array}{l}-2.621^{*} \\
(1.405)\end{array}$ & $\begin{array}{c}0.483 \\
(1.107)\end{array}$ & $\begin{array}{l}-0.122 \\
(1.276)\end{array}$ \\
\hline $\begin{array}{l}\text { B_Dissimilarity of Local Policy } \\
\text { (Consumer Price Index differential) }\end{array}$ & & & & & $\begin{array}{c}-0.082 * * * \\
(0.019)\end{array}$ & $\begin{array}{c}-0.065 * * * \\
(0.021)\end{array}$ & $\begin{array}{c}-0.069 * * * \\
(0.021)\end{array}$ & $\begin{array}{c}-0.062 * * * \\
(0.021)\end{array}$ & $\begin{array}{c}-0.058 * * \\
(0.025)\end{array}$ \\
\hline C_ Railways Freight Traffic (2) & & & & & & $\begin{array}{l}0.654^{* *} \\
(0.270)\end{array}$ & $\begin{array}{l}0.480 * \\
(0.278)\end{array}$ & $\begin{array}{l}0.425^{*} \\
(0.253)\end{array}$ & $\begin{array}{c}0.620 * * \\
(0.305)\end{array}$ \\
\hline D_ Dissimilarity of Trade Intensity & & & & & & & & $\begin{array}{l}-0.052 \\
(0.105)\end{array}$ & $\begin{array}{l}-0.106 \\
(0.110)\end{array}$ \\
\hline E_ Dissimilarity of International Partner & & & & & & & $\begin{array}{c}-0.407^{* *} \\
(0.195)\end{array}$ & & $\begin{array}{c}-0.500^{* *} \\
(0.231)\end{array}$ \\
\hline Constant & $\begin{array}{c}0.671 * * * \\
(0.016)\end{array}$ & $\begin{array}{c}0.809 * * * \\
(0.036)\end{array}$ & $\begin{array}{c}1.117 * * * \\
(0.055)\end{array}$ & $\begin{array}{c}1.173^{* * *} \\
(0.063)\end{array}$ & $\begin{array}{c}1.308 * * * \\
(0.072)\end{array}$ & $\begin{array}{c}1.131 * * * \\
(0.073)\end{array}$ & $\begin{array}{c}1.400 * * * \\
(0.112)\end{array}$ & $\begin{array}{c}1.123 * * * \\
(0.089)\end{array}$ & $\begin{array}{c}1.306 * * * \\
(0.113)\end{array}$ \\
\hline Observatins & 378 & 378 & 378 & 378 & 378 & 378 & 378 & 378 & 378 \\
\hline $\mathrm{R}^{2}$ & 0.078 & 0.16 & 0.28 & 0.26 & 0.28 & 0.13 & 0.14 & 0.27 & 0.09 \\
\hline Hansen Overindentification Test & & & & 0.21 & $0.14 \mathrm{~S}$ & 0.26 & 0.22 & 0.14 & 0.78 \\
\hline $\begin{array}{l}\text { List of Endogenous Variables (partial } \mathrm{R}^{2} \\
\text { of excluded instruments in parentheses) }\end{array}$ & & & & $\mathrm{A}(0.21)$ & $\mathrm{A}(0.22)$ & $\begin{array}{l}\text { A }(0.31) \text { and } \\
\text { C }(0.10)\end{array}$ & $\begin{array}{c}\mathrm{A}(0.38), \mathrm{C} \\
(0.13) \text { and } \mathrm{E} \\
(0.23)\end{array}$ & $\begin{array}{c}\text { A (0.44), C } \\
(0.10) \text { and D } \\
(0.21)\end{array}$ & $\begin{array}{c}\mathrm{A}(0.46), \mathrm{C} \\
(0.10), \mathrm{E}(0.21) \\
\text { and D }(0.24)\end{array}$ \\
\hline
\end{tabular}

Robust standard errors in parentheses calculated as indicated by White (1980)

* significant at $10 \%$;* significant at $5 \%$; ** significant at $1 \%$ 
interior provinces that for the greatest part of their history have been disadvantaged by both their inferior conditions for economic growth in a large sense and by neglect or even discrimination on the part of the central government (Lin et al., 2002; Démurger et al., 2002).

A lower correlation between provinces could be the consequence of high average distance between them. We follow Clark and van Wincoop (2001) and assume that business cycle correlation reflects interaction intensity. As such we take into account various structural exogenous elements in the analysis of business cycles' correlation between Chinese provinces. We control for the distance separating the provinces as well as for their potential adjacency. Respectively negative and positive signs are anticipated as we expect lower correlation to prevail between further apart provinces (Clark and van Wincoop, 2001, Barrios et al., 2003). Moreover as higher correlation between provinces could be the result of their larger sizes and therefore greater opportunities for within-province diversification, we incorporate a variable that captures the combined size. We expect the size effect to be positive (Clark and van Wincoop, 2001; Barrios and de Lucio, 2003; Barrios et al., 2003).

\section{Empirical Results}

Results are reported in Table 3, 4 and 5 for each of the three sub-periods successively. They follow the same logical order in terms of explanatory variables introduction. In addition to exogenous spatial characteristics, we successively assess the role of four main factors: sectoral specialization dissimilarity, local policy coordination, domestic integration, as well as differences in terms of international trade engagement.

Various tests enable us to control for the quality and reliability of our empirical strategy. At the bottom of the results tables, we successively report the explanatory power of the regression, the Hansen $\mathbf{J}$ statistic, the list of endogenous variables and the list of instruments used in the IV procedure. We further report the partial $\mathrm{R}^{2}$ of excluded instruments in the case of 2-SLS estimation. It provides the explanatory power of instruments for each endogenous variables. The Hansen Sargan test of over-identifying restrictions helps checking that instrumental variables are valid (i.e. uncorrelated with the error term) and that the excluded instruments are correctly excluded from the estimated equation. Under the null hypothesis, the test statistic is distributed as chi-squared in the number of over-identifying restrictions. 
A rejection casts doubt on the validity of the instruments. In no case the validity of our instruments is rejected.

\section{A. Identification of Lines of Fracture and Control for Exogenous Factors}

In Column 1 of these tables, we focus on the potential cleavage existing between Interior and Coastal parts of China. We rely on two complementary dummy variables that capture the divergence from the benchmark that is the average business cycle correlation between interior provinces. The first dummy (Intra Coast) equals 1 when provinces $i$ and $j$ are located in the Coastal region and 0 otherwise. It measures the extent to which average synchronization betwee Coastal provinces differ from that between interior provinces. The second dummy (InteriorCoast border) equals 1 when provinces $i$ and $j$ are located across the border separating the interior and coastal regions and 0 otherwise.

Over the first sub-period the intra-Coast dummy enters with a positive and significant sign while the Interior-Coast border dummy fails to be significant. This result indicates that interior provinces are characterized by lower average synchronization between each other in the beginning of the 1990s. There is a radical change between the first and the subsequent periods. While over the first half of the 1990s coastal provinces appear to enjoy greater economic correlation than the rest of the country, they subsequently display lower synchronization than that taking place between interior provinces. One feature is worth noticing: over the first two sub-periods, the significance of the two regional dummies fades away when dissimilarities of international trade intensity are accounted for (columns 79). It suggests that the lack of economic cohesion in China is rooted in this international trade heterogeneity. However, over the last period, both dummies remain significant. This last result highlights that economic fragmentation in China derives from the regionally differentiated economic policies that go beyond international openness. The negative and significant coefficient on Interior-Coast border signals that correlation between interior provinces exceeds that between coastal provinces as well as that between coastal and interior.

Column 2 (Tables 3, 4 and 5) reports the results when Size, Distance ${ }^{10}$ and Adjacency variables are added in the regression of business cycle correlations beside intra-Coast and Interior-Coast dummies. Size $e_{i j}$ is measured as the product of

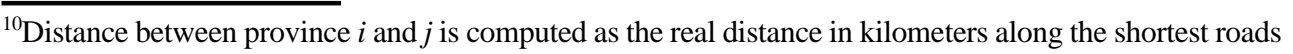
between $i$ 's and $j$ 's capital cities. Details are provided in the appendix. 
provincial populations averaged over the relevant estimation period. Adjacency is a dummy variable that equals 1 when regions $i$ and $j$ are contiguous and 0 otherwise. It intends to capture greater correlation deriving from privileged links existing between directly connected provinces. As expected, results confirm that higher correlation is observed for physically closer provinces. ${ }^{11}$ There is no significant effect for being adjacent provinces. Findings of a significant impact of distance, size, and adjacency that are also significant determinants of trade flow dynamics in China (Poncet, 2005) suggest that flows of goods between provinces would be relevant to understand regional co-movement of economic activity.

\section{B. Influence of Regional Output Specialization}

Column 3 in all three tables introduces, parallel to exogenous factors presented above, an indicator of regional output specialization, to determine whether business cycles' in China are closer for provinces with more similar production structures.

Kenen (1969) and Krugman (1993) stress that in the presence of industryspecific shocks a higher similarity in production structures across regions is likely to be associated with greater business cycles' correlation, as two economies producing the same types of goods will be subject to similar stochastic developments. In line with Kraay and Ventura (2001)'s findings, Imbs (2003) underlines that in a context of aggregate shocks, business cycles' in countries with similar production patterns will be synchronized in case sectors differ in their response to shocks, for instance because of different market structures or labor market arrangements. Empirical evidence of the positive relationship of structure similarity on business cycles' synchronization is found in analyses using regional data (Kalemli-Ozcan et al., 2001; Clark and van Wincoop, 2001; Barrios and de Lucio, 2003; Barrios et al., 2003) as well as national data (Imbs, 2003; Calderón et al., 2003).

Following Clark and van Wincoop (2001), we rely on a measure of structural dissimilarity proposed by Krugman (1991): production structure dissimilarity $=\sum_{k=1}^{n}\left|S_{i k}-S_{j k}\right|$, where $S_{i k}$ and $S_{j k}$ denote respectively the average share of activity $k$ in province $i$ and $j$ 's GDP. Note that the higher the index value, the greater the difference in industry shares across provinces $i$ and $j$ and therefore, the greater the differences in economic structure. Our measure covers 24 sectors for all Chinese

\footnotetext{
${ }^{11}$ Results for the sub-period 1996-2000 however contrast with this expected finding. They suggest a counterintuitive positive and positive impact of distance on business cycles correlation.
} 
provinces. ${ }^{12}$ We expect to observe a strong relationship between the degree of specialization across those broad sectors and the degree of regional co-movement, if, as suggested by Long and Plosser (1983), business cycles' are driven by specific shocks to broad industries.

As argued by Kalemli-Ozcan et al. (2001), we cannot a priori rule out that specialization is unaffected by fluctuations asymmetry. A province may attempt to reduce the size of a sector in which activity is characterized by a high variance relative to other provinces for reasons unrelated to the industrial structure. This scenario is however not validated, since the Hausman test of exogeneity does not reject the hypothesis of good performance of OLS.

Results in column 3 suggest a negative and significant impact of production dissimilarities on the synchronization of business cycles' of Chinese provinces. This impact appears to be especially strong during the second half of the 1990s in coherence with provincial authorities' engagement in industrial policy planning during the Ninth Year Plan. The detrimental influence of specialization on business cycle correlation turns out to be particularly robust as it persists after other policy determinants are introduced in the regression from 1995 onwards. Over the first period, however, the impact of production dissimilarities becomes insignificant when the freight traffic variable is included. This result is coherent with a positive correlation for that period between freight traffic and production structure differentials.

Findings of this section suggest that the ongoing increase in regional specialization of China's industries (Bai et al., 2004) amplifies output fluctuations of Chinese provinces. Results also underline that Chinese authorities can enhance the synchronization of business cycles by promoting domestic trade, possibly through investments in transport infrastructure.

\section{Influence of Economic Policy Coordination}

Local policy coordination is also traditionally considered as a key determinant of business cycles' synchronization (Fatás, 1997). A greater coordination of economic policies, however, has an ambiguous impact on business cycles' since it depends on the type of shocks driving economic fluctuations and on the ability to stabilize output. If macroeconomic policies are themselves a source of business cycles', more coordinated policy could then lead to higher synchronization of economic

${ }^{12}$ Data are taken from the 1992 provincial input output tables. 
fluctuations. However, common policies could reduce local governments' flexibility to dampen regional business cycles', which can result in less coordinated business cycles'. As this issue is ultimately an empirical one, we examine the impact of including two indicators of local policy similarity in our previous model. They intend to capture provincial divergence in terms of fiscal policy and of global economic autonomy respectively.

Similarly to Clark and Van Wincoop (2001), we investigate the prevailing relationship between fiscal coordination and business cycle synchronization in the context of China based on the standard deviation of provincial budget deficit differentials. We rely on annual budget deficit data expressed as a percentage of GDP over the period from 1990 to 2002.

We follow Boyreau-Debray (2000) and consider provincial heterogeneity in terms of inflation to reveal local autonomy patterns. This author finds that provincial CPI divergence from the national average is directly associated with local policy dissimilarities that go beyond fiscal practices to include differences in terms of firm ownership and economic interventionism. We compute average absolute difference of consumer price index to proxy local autonomy difference.

We rely on $\frac{\sum_{t}\left|I P C_{i, t}-I P C_{j, t}\right|}{t}$ where $I P C_{i, t}$ is the consumer price index of the province $i$ during the period $t$. We compute this average index over the six year period preceding the sub-period for which business cycle correlation is computed (i.e. 1986-1991, 1990-1995 and 1995-2000).

We deal with the potential endogeneity of these policy difference measures: larger asymmetric shocks can lead to larger differences in local policy. Estimations are run using the two-stages-least-squares (2SLS) procedure. ${ }^{13}$

Results are reported in columns 4 and 5. Both indicators of local policy dissimilarity ${ }^{14}$ enter with a negative sign in all regressions, suggesting that the greater local policy divergence, the lower business cycle correlation. Provinces conducting similar local policy tend to have more synchronized business cycles'.

\footnotetext{
${ }^{13}$ Our indicator of bilateral deficit standard deviation turns out to be endogenous for all sub-periods. Our main instrument is the joint existence of an international border (that takes the value of 1 when one of the province shares a land or sea frontier with an international country, 2 when both provinces do and 0 otherwise. Our instrument list includes the difference between the two provinces in terms of population density and primary GDP as well as the sum of the two provinces' value in terms of urbanization rate and of the share of population in the primary sector.

${ }^{14}$ To test the robustness of these results, we also use another indicator of fiscal policy coordination. Bilateral deficit correlation coefficient entered as expected by theory with a significant positive sign.
} 
Table 6. Summary Statistics of Variables

\begin{tabular}{lccc}
\hline \multicolumn{1}{c}{ Variable } & & Mean & $\begin{array}{c}\text { Standard } \\
\text { Deviation }\end{array}$ \\
\hline & & .400 & .231 \\
Output Correlation & $1991-1995$ & .204 & .340 \\
& $1996-2000$ & .579 & .252 \\
Intra Coast & $2001-2004$ & .146 & .353 \\
Coast-Interior Border & & .495 & .501 \\
Size ${ }^{1)}$ & & .186 & .176 \\
Distance ${ }^{2)}$ & & .206 & .109 \\
Adjacency & & .172 & .378 \\
Dissimilarity of Production Structure & & .642 & .198 \\
Dissimilarity of Local Policy & & .029 & .0218 \\
(standard deviation of public deficit) & & & \\
& & 2.280 & 1.314 \\
Dissimilarity of Local Policy & $1991-1995$ & 2.711 & 1.068 \\
(Consumer Price Index differential) & $1996-2000$ & 1.575 & .577 \\
& $2001-2004$ & .065 & .159 \\
Railways Freight Traffic (2) & $1991-1995$ & .076 & .185 \\
& $1996-2000$ & .085 & .217 \\
Dissimilarity of International Trade Intensity & $2001-2004$ & .141 & .163 \\
Dissimilarity of International Partner & $1991-1995$ & .223 & .300 \\
\hline Notes: & $1996-2000$ & .348 & .403 \\
\hline
\end{tabular}

Notes : 1) and 2): coefficients have been multiplied by $10^{10}$ and $10^{4}$ respectively

This feature gives support to the view of local policy as a source of fluctuations and not as a stabilizer of business cycles'. While over the 1900-1995 period, only fiscal policy divergence enters significantly, both proxies for the lack of policy coordination subsequently turn out to have a significant detrimental effect on economic cohesion.

Results highlight that fiscal autonomy as well as broader economic interventionism act as two distinct and complementary sources of destabilization. The significant reduction in the coefficient in front of the fiscal policy differentials over the most recent period seems to indicate that the 1994 fiscal reform was quite successful in mitigating fiscal decentralization and in promoting fiscal rationalization. Notably, we estimate that over the period 2001-2004, a one standard deviation increase in fiscal divergence between a pair of provinces induces a $10 \%$ reduction in their business cycles' correlation. This effect is both 
Table 7. Impact of One Standard Deviation Increase over the 2001-2005 sub-period

\begin{tabular}{lc}
\hline \multicolumn{1}{c}{ Variables } & $\begin{array}{c}\text { Range of Impact } \\
\text { (coefficient*standard deviation of } \\
\text { explanatory variable) }\end{array}$ \\
\hline Size & $-0.030 /-0.056$ \\
Distance & $-0.036 /-0.073$ \\
Dissimilarity of Production Structure & $-0.085 /-0.129$ \\
Dissimilarity of Local Policy & $-0.057 /-0.066$ \\
(standard deviation of public deficit) & \\
Dissimilarity of Local Policy & $-0.033 /-0.047$ \\
(Consumer Price Index differential) & \\
Railways Freight Traffic (2) & $0.092-0.142$ \\
Dissimilarity of International Partner & $-0.098 /-0.121$ \\
\hline
\end{tabular}

Chinese average of output correlation over the same sub-period : 0.58

statistically and economically significant. Such a computation is based on the multiplication of the coefficient value and the standard deviation of fiscal divergence across provinces. Summary statistics of variables are reported in Table 6 while estimated impacts for all explanatory indicators appear in Table 7. The impact of one standard deviation increase in the explanatory variable has to be put in line with the average output correlation over the period.

As far as policy implications are concerned, our results indicate that stabilization of output fluctuations can be achieved in China by promoting further coordinating fiscal and economic policies in the provinces.

\section{Influence of Domestic Economic Integration}

The influence of market integration on business cycles' synchronization received much attention especially in connection with the debate on the European Monetary Union. The objective of most of studies in the literature was to determine whether and which EU countries would form a viable and beneficial unique currency area in light of the existing (ex ante) economic integration between them. Here, our intention is to verify whether we find Chinese provinces to have more correlated business cycles' when they are similar in terms of domestic trade. The results will carry policy implications on how to promote deeper integration in China.

As synthesized by Calderón et al. (2002), the impact of trade integration on business cycles' correlation could in fact go either way. On the one hand, if the demand channel is the dominant force driving business cycles', the more intense 
bilateral trade is expected to accompany highly correlated business cycles' (Imbs, 2003 and 2004). As an illustration, let a country increase its demand for outside goods following a positive output shock. The impact of this shock on the cycle of the country's trading partners should depend on the trade flows intensity between them. On the other hand, if industry-specific shocks are the dominant force in explaining cyclical output, the relationship could be reversed in case increasing specialization in production translates into inter-industry trade (as predicted in standard trade theory like the Heckscher-Ohlin paradigm). Trade integration may lead to increased specialization of each country on their (different) comparative advantages, resulting in more pronounced business cycles' due to asymmetric effects of industry-specific shocks (Eichengreen, 1992; Krugman, 1993). If the case were that the patterns of specialization are mainly intra-industry (as observed in industrial countries) trade integration would not necessarily lead to greater asymmetry. To summarize, the question of the total effect of trade intensity on business cycle correlation is to be answered empirically. The positive influence of trade in goods on the degree of business cycles' synchronization is in fact a robust finding across empirical studies (Clark and van Wincoop, 2001; Frankel and Rose, 1998; Calderón et al., 2003). ${ }^{15}$

Frankel and Rose (1998) demonstrate that trade integration significantly raises business cycle synchronization. Furthermore, Baxter-Kouparitsas (2005) stress that among the various candidate determinants of business cycle synchronization suggested in the literature, only trade integration has a robust effect.

Unfortunately, no data exists on bilateral trade flows between Chinese provinces, preventing us from looking at the influence of bilateral trade integration on business cycle co-movement. ${ }^{16}$ The only available series that we are aware of provides the freight traffic volume through the railway system between and within provinces that evidently is only a partial measure of total trade.

It is important to stress that railway freight volume is a truly imperfect proxy for domestic trade, especially in the preliminary stage of the reforms. This is due to the

\footnotetext{
${ }^{15}$ More importantly, Clark and Van Wincoop (2001) show that incorporating trade into the regression equations turns the border effect that distinguishes between intra-national and international fluctuations insignificant. This finding indicates that higher levels of within-country trade can explain most of the observed differences between within-country and cross-country business cycle correlations.

${ }^{16}$ The availability of Census data on bilateral migration flows between provinces induced us to test the potential impact of labor mobility on business cycle correlation. Our migration indicator failed to enter significantly in our model. This may be due to the fact that migration flows are a poor proxy of labor dynamics due to widespread regional migration restrictions and measurement errors.
} 
very partial product coverage and heterogeneous spatial density of the railway transportation mode. Although coal, grain, iron and steel account for well over half of railroad freight volume, they make up at most ten or fifteen percent of the value of inter-provincial trade.

The use of railway freight volume as an indicator of trade integration is especially problematic for the first sub-period of our sample. We should consider these points from Rong, Li et al. (1997):

"The inadequacy of the national transport system remains the major barrier to greater regional integration... Coal alone accounts for two-fifths of the overall railway ton $/ \mathrm{km}$ freight task... Apart from coal, the other main freight flows are of grain and iron and steel (each 8 per cent of the railway ton $/ \mathrm{km}$ task)... The main transport problem... is the serious shortage of railway capacity, largely because of the priority given to coal, building materials, and other bulk freight. Space for nonpriority freight has to be booked weeks in advance and there is no certainty as to when consignments will reach their destination."

As emphasized by Naughton (2003), over the beginning of the 1990s, "the need to ensure shipments of these bulk commodities obstructed the development of shipments of high value commodities. However, that is unlikely to be the case today, except in individual areas of China”. Indeed, during the 1990s, China made a major investment effort to upgrade its transportation and communications infrastructure. During the eighth Five-Year Plan period (1991-1995), as put forward by officials, successes in building up basic industries and infrastructure eased the "bottleneck" constraints on economic growth, providing additional strength for future economic development. As such we expect that railway freight volume correlates more closely to total trade integration towards the end of the $20^{\text {th }}$ and beginning of the $21^{\text {st }}$ century.

We analyze the role of freight traffic as a determinant of business cycles' synchronization based on the average sum of freight traffic. Freight Traffic ${ }_{i j}=$ $\left(\right.$ Freight Traffic $_{i j}+$ Freight Traffic $\left.{ }_{i j}\right) / 2$ is computed over the relevant sub-periods. Endogeneity is likely to be a problem since higher business cycle synchronization may result in stabilized and large bilateral trade. Column 6 in Tables 3 to 5 reports 2SLS estimation results. ${ }^{17}$ Freight traffic turns out to have an unexpected negative

\footnotetext{
${ }^{17}$ Our indicator of domestic trade intensity is instrumented using the joint distance to the national capital city as well as the absolute difference between the two provinces in terms of their domestic remoteness defined following Helliwell's (1997): Remote Re $_{i} \sum_{k \neq i, j} \frac{\text { Distance }_{i k}}{G D P_{k}}$.
} 
and significant impact on business cycles' synchronization over the first subperiod. Its influence becomes positive and significant over the two subsequent subperiods 1996-2000 and 2001-2004. This evolution is coherent with the drastic improvement of transportation infrastructure over the 1990s. Over the sub-period, freight traffic may act as a proxy of bottlenecks and irrationality of the allocation system while it better corresponds to the internal trade intensity. Results attest to the beneficial impact of domestic integration from mid 1990s onwards. They are coherent with the view that global shocks dominate economic fluctuations or that industry shocks lead to vertical specialization. These results, in contrast, refute the view that industry shocks are dominant. They invalidate the Heckscher-Ohlin prediction that trade openness leads to complete specialization. We can compute that, over the period from 2001 to 2004, a one standard deviation increase in freight traffic intensity between two provinces induces a $15 \%$ increase in their business cycles' correlation. Over that sub-period, our proxy of domestic trade integration turns out to be the strongest force behind business cycles' synchronization.

Our results confirm the policy implications presented in section B that the synchronization of business cycles can be improved by promoting domestic trade, possibly through investments in transport infrastructure and though tougher stand against provincial domestic trade protection.

\section{E. Influence of Dissimilarity of International Economic Integration}

As a last step of our research, we investigate the influence of the growing heterogeneity between Chinese provinces in terms of international trade openness. Our approach focuses on differences in international trade flows intensity and partners.

When considering the influence of the disparity between provinces' international trade intensity, we are interested in determining whether the heterogeneity in the degree of international economic integration between two provinces has an implication for the synchronization of their business cycles'. We would expect similar international trade intensity for two provinces to induce greater economic business cycles' synchronization between them as they are influenced to a similar extent by the international cycle. Under this scenario, similar levels of international trade for two provinces would induce tighter economic business cycles' between them. Moreover, as noted by Kalemli-Ozcan et al. (2001), higher integration in international markets would enable provinces to insure themselves against 
asymmetric shocks through diversification of ownership and therefore allow them to engage into more specialized production structure. In this case, diverging opportunities for income diversification due to dissimilar international integration are expected to be associated with more asymmetric business cycles'. An alternative scenario could, however, be that international trade acts as a stabilizing force and facilitates regional adjustment to idiosyncratic shocks, especially in a context of poor domestic integration. Under this hypothesis, diverging degrees of international integration would be associated with stronger business cycle correlation.

We rely on an indicator that contrasts the international openness degree of provinces $i$ and $j$. We calculate International trade intensity dissimilarity $i_{i j}=\left|F T_{i}-F T_{j}\right|$ where $F T_{i}$ and $F T_{j}$ correspond to the average ratio of trade to GDP of provinces $i$ and $j$ respectively.

We anticipate that the impact of international trade intensity dissimilarity differs depending on the time period. At a primary stage of international openness and reforms, heterogeneous international trade ratios could correspond to heterogeneous needs for adjustment to idiosyncratic shocks across provinces. Subsequently, with the rapid liberalization of international flows, the extent to which international trade intensity differs across China rises dramatically (see Table 7), possibly to a point where it is associated with lower business cycle correlation between provinces that are very much integrated with the international market and those that are mainly dependent on the domestic market.

Aside from differences in terms of international trade intensity, we also investigate the influence of differences in terms of partners. We compute an indicator of partner dissimilarity derived from Krugman (1991)'s formula for output structure dissimilarity, defined as International Partner dissimilarity ${ }_{i j}$ $=\sum_{p=1}^{n}\left|S_{i p}-S_{j p}\right|$, where $S_{i p}$ and $S_{j p}$ denote the share of partner $p$, respectively inprovince $i$ and $j$ 's international export flows.

We would expect great inter-provincial disparity in terms of international partner structure to correspond to high heterogeneity in terms of influence by different international cycles'. Under this scenario, similar partner structures for international trade for two provinces would induce tighter economic business cycles' between them. Columns 7-9 report results including dissimilarity of international partner and trade intensity relying on 2SLS. One may suspect our international trade intensity dissimilarity and international partner dissimilarity indicators to be endogenous as trade policy can be influenced by the magnitude of 
business cycles' ${ }^{18}$

Results emphasize the negative and significant impact of inter-provincial differences in terms of international trade intensity over the sub-period 1996-2000 while dissimilarity in terms of trade partners fails to enter significantly. Findings are reserved over the last sub-period. From 2000 onwards, inter-provincial disparity in terms of international partners becomes the strongest detrimental force to business cycle correlation.

As far as policy implications are concerned, our results indicate that stabilization of output fluctuations can be achieved in China by promoting more homogenous trade openness across provinces, possibly though better information and network sharing.

\section{Conclusion}

This paper documents business cycles' fluctuations in China. On the basis of output data in Chinese provinces, we emphasize that cycles' synchronization increased from a rather low level at the beginning of the 1990s to a level comparable to that of the US at the beginning of the 2000s. Results stress the cleavage existing between the coastal and interior regions: business cycles' correlation between coastal regions appears to be much lower than that in the rest of the country. In that sense the Chinese market appears to be split into two parts. Our investigation of the role that various potential domestic determinants play in the synchronization of business cycles' is conclusive in the context of the Chinese economy. Trade integration and fiscal and economic policy coordination are found to foster business cycles' synchronization. On the other hand, production structure divergence and international trade dissimilarity tend to reduce business cycles' comovements. As far as policy implications are concerned, our results indicate that stabilization of output fluctuations can be achieved in China by promoting domestic trade and further coordinating fiscal and economic policies in the provinces.

\footnotetext{
${ }^{18}$ Both indicators of international trade heterogeneity are instrumented using the absolute difference between the two provinces in terms of their international remoteness defined as

$\sum_{k \text { foreign partner }} \frac{\text { Distance }_{i k}}{G D P_{k}}$ as well as their difference in terms of surface area and their joint ratio of highway length on surface area.
} 


\section{Data Sources}

Monthly industrial output data in $\mathbf{1 9 9 0}$ yuan price at the provincial level are taken from the China Monthly Statistics edited by China Statistical Information and Consultancy Service Center under the National Bureau of Statistics.

Distance: Bilateral distances between provinces are measured on the basis of real distance by road in kilometers between their capital cities following the quickest route based on very detailed maps. This measure, which takes into consideration the reality of geographical space (mountains. lakes. density and quality of road infrastructures), is a better proxy than Great Circle distance that is generally used in economic geography or trade studies.

Production structure: A broader decomposition is available for 1992 from the China Industrial Economic Statistical Yearbook (State Statistical Bureau). We rely on gross output data. A total of 23 industrial sectors are covered from Beverage manufacturing to Power generation.

Domestic Trade: Data on freight traffic through railways (in tons) between provinces are taken from China transportation and communications yearbook. These statistics are imperfect representative of the total trade flows of provinces since the railways freight traffic volume is very much dependent on the alternative means of transportation available in the province. The main alternatives of transportation are highways and waterways. We therefore by no means pretend to measure domestic trade as a whole but rather a share of it, knowing that the size of this share is very heterogeneous across provinces.

International trade partner structure: We rely on the decomposition of provincial exports for 224 international partners obtained from Chinese Customs for the year 1998.

\section{References}

Bai, C-E., Du, Y., Tao, Z., Tong, S. Y. (2004), "Protectionism and Regional Specialization: Evidence from China's Industries", Journal of International Economics, 63 (2), p. 397-417.

Bahl R. (1999), "Fiscal Policy in China, Taxation and Intergovernmental Fiscal 
Relations", The 1990 Institute, pp. 236.

Barrios, Salvador and de Lucio, Juan J. (2003), "Economic Integration and Regional Business Cycles": Evidence from the Iberian Regions", Oxford Bulletin of Economics and Statistics, 65 (4), pp.497-515.

Barrios, Salvador, Brülhart, Marius., Elliot, Robert., and Sensier, Marianne. (2003). "A Tale of Two Cycles: Co-fluctuations between UK Regions and the Euro Zone", The Manchester School, 71 (3), pp. 265-292

Baxter, Marianne and Michael A. Kouparitsas (2005), "Determinants of Business Cycle Comovement: a Robust Analysis", Journal of Monetary Economics., 52, pp.113-157.

Boyreau-Debray, Geneviève. (2000), "Politique économique locale et inflation en Chine", Revue Economique, 51 (3), pp. 713-724.

Bystrom, Hans N.E., Olofsdotter, Karin, and Lars Soderstrom (2005), Journal of Asian Economics, Volume 16, Issue 4, pp. 612-634

Calderón César, Chong Alberto and Stein Ernesto (2003), "Trade Intensity and Business Cycle Synchronization: Are Developing Countries Any Different?” Working Paper 478, IADB, Research Department.

Chen, J., and Fleisher, B. -M. (1996), "Regional Income Inequality and Economic Growth in China", Journal of Comparative Economics, 22, 141164.

Clark, Todd and van Wincoop, Eric (2001), "Borders and Business Cycles", Journal of International Economics, 55 (1), pp. 59-85.

Démurger Sylvie, Jeffrey D. Sachs, Wing Thye Woo, Shuming Bao, Gene Chang and Andrew Mellinger (2002), "Geography, Economic Policy and Regional Development in China", in Asian Economic Papers, Vol. 1, No. 1, pp. 146-197.

Donnithorne, Audrey (1972), "China's Cellular Economy: Some Trends since the Cultural Revolution”, China Quarterly, 52, pp. 605-619.

Eichengreen, Barry (1992), "Is Europe an Optimum Currency Area?" in Grubel, H., Borner S. (Eds), The European Community after 1992: Perspectives from the Outside. MacMillan, Basingstoke, England, pp. 138-161.

Fatás, Antonio (1997), "EMU: Countries or Regions? Lessons from the EMS Experience", European Economic Review, 41, (3-5), pp. 743-751.

Frankel, Jeffrey A. and Rose, Andrew (1998), "The Endogeneity of the Optimum Currency Area Criteria", Quarterly Journal of Economics, 108, pp. 1009-25.

Hausman Jerry A. (1978), "Specification Tests in Econometrics", Econometrica 50, May, pp 749-759

Hodrick, Robert J., and Prescott, Edward (1997), "Postwar U.S. Business Cycles: an Empirical Investigation", Journal of Money, Credit, and Banking, 29, pp. 1-16.

Hua Ping and Sylviane Guillaumont-Jeanneney (2004), "Why Do More Open Chinese Provinces Have Bigger Governments?" Review of International Economics, Vol. 12, No. 3, pp. 525-542.

Imbs, Jean and Romain Wacziarg (2003), "Stages of Diversification", American Economic Review, 93(1), pp. 63-86

Imbs, Jean (2003), Co-fluctuations, Revised Version of Imbs, J., (1998), DEEP Working 
Paper 98-19, University of Lausanne.

Imbs, Jean (2004), Growth and Volatility, Revised Version of Imbs, J. (2002), CEPR Discussion Paper 3561.

Jian, T., Sach, J. -D., \& Warner, A. -M. (1996), "Trends in Regional Income Inequality in China", NBER Working Paper, vol. 5412.

Kalemli-Ozcan Sebnem, Bent E. Sørensen, Ariell Reshef and Oved Yosha (2001), "Economic Integration, Industrial Specialization, and the Asymmetry of Macroeconomic Fluctuations", Journal of International Economics, 55, pp. 107-137

Kenen, Peter (1969), "The Theory of Optimum Currency Area: an Eclectic View", in: Mundell, R.,

Kraay, Aart and Ventura, James (2001), "Comparative Advantage and the Cross-section of Business Cycles", NBER Working Paper 8104.

Krugman, Paul (1991), "Geography and Trade," MIT Press, Cambridge.

Krugman, Paul (1993), "Lessons from Massachusetts for EMU", in Torres, F. and Giavazzi, F. (Eds.), Adjustment and Growth in the European Monetary Union, Cambridge University Press, Cambridge, 37

Lin, Justin Yifu, Cai Fang, and Li Zhou (2002), "Social Consequences of Economic Reform in China: an Analyses of Regional Disparity in the Transition Period", in Mary-Françoise Renard (ed.) China and its Regions - Economic Growth and Reform in Chinese Provinces, Cheltenham/Northampton: Edward Elgar.

Long, John B. and Plosser, Charles (1983), "Real Business Cycles", Journal of Political Economy, 93, pp. 39-69.

Ma Jun and John Norregaard, (1998), "China's Fiscal Decentralization", IMF, Unpublished Manuscript.

Mundell Robert A. (1961), "A Theory of Optimum Currency Areas", American Economic Review, 51(4), pp. 657-665

Naughton, Barry (2003), "How Much can Regional Integration Do to Unify China's Markets?", in Nicholas Hope, Dennis Yand and Mu Yang Li (eds.), How Far across the River? Chinese Policy Reform at the Millenium, Stanford: Stanford University Press, pp. 204-232.

Narayan P. K. (2008), "Evidence of Panel Stationarity from Chinese Provincial and Regional Income", 19, p. 274-286.

Poncet Sandra (2005), "A Fragmented China: Measure and Determinants of Chinese Domestic Market Disintegration", Review of international Economics, 13 (3), pp. 409-430.

Ravn Morten Overgaard and Uhlig Harald (2002), "On Adjusting the HP-filter for the Frequency of Observations", Review of Economics and Statistics, 84 (1), pp. 371-80.

Rong Chao-he, Li Wen-yan, Linge, Godfrey and Forbes, Dean K. (1997), "Linking the Regions: a Continuing Challenge", p. 46-71 in China's New Spatial Economy: Heading Towards 2020. ed. Godfrey Linge. Hong Kong, Oxford University Press.

Sakamoto H. and N. Islam (2008), "Convergence across Chinese Provinces: an Analysis using Markov Transition Matrix”, 19 (1), p. 66-79. 
Shin Kwanho and Wang Yunjing (2004), "Trade Integration and Business Cycle Synchronization in East Asia", Asian Economic Papers 2 (3), pp. 1-20.

White, Halbert L. (1980), "A Heteroskedasticity-consistent Covariance Matrix Estimator and Direct Test for Heteroskedasticity", Econometrica, 48 (4), pp. 817-38.

Yang, Dali L. (1997), "Beyond Beijing: Liberalization and the Regions in China", Routledge.

Young, Alwyn (2000), “The Razor's Edge: Distortions and Incremental Reform in the People's Republic of China", Quarterly Journal of Economics, 115, pp. 1091-1136.

Zhang, Tao and Zou Heng-fu (1998), "Fiscal Decentralization, Public Spending and Economic Growth", Journal of Public Economics, 67 2, pp. 221-240. 